\title{
Filigrane
}

Écoutes psychanalytiques

\section{L'assignation de genre et le sexuel infantile}

\section{Dominique Scarfone}

Volume 28, numéro 1, 2019

Identités $^{2}$. Qui suis-je ?

URI : https://id.erudit.org/iderudit/1064595ar

DOI : https://doi.org/10.7202/1064595ar

Aller au sommaire du numéro

Éditeur(s)

Santé mentale et société

ISSN

1192-1412 (imprimé)

1911-4656 (numérique)

Découvrir la revue

Citer ce document

Scarfone, D. (2019). L’assignation de genre et le sexuel infantile. Filigrane, 28(1),

33-42. https://doi.org/10.7202/1064595ar d'utilisation que vous pouvez consulter en ligne.

https://apropos.erudit.org/fr/usagers/politique-dutilisation/ 


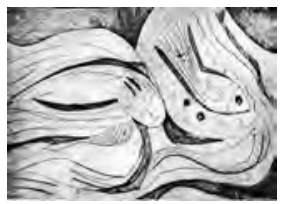

\section{L'assignation de genre et le sexuel infantile ${ }^{1}$}

\section{Dominique Scarfone}

Cher Jacques,

Sans surprise, je me suis retrouvé tout de suite dans le paysage que tu nous offres depuis des décennies, fait de rigueur du contenu, d'élégance du style, d'appel aussi à la sobriété psychanalytique. Les idées que tu avances sont claires et affirmées à partir d'une base clinique et théorique solide, comportant pour moi des repères qui, pour être familiers, n'en sont pas moins essentiels et toujours à reprendre. On sait que pour la psychanalyse la familiarité est sœur du refoulement, et c'est ce qui fait que chaque lecture d'un texte métapsychologiquement bien fondé fait œuvre de levée de ces petits refoulements qui finissent par assoupir la pensée. Ton texte a eu sur moi cet effet, et $j$ 'espère aussi sur tes auditeurs. Le commentaire qui suit est donc une sorte de vérification ou de mise à l'épreuve de cette levée du refoulement qui nous est périodiquement nécessaire.

Je ne saurais, évidemment, reprendre ton texte point par point. Je me concentrerai sur certains aspects qui me semblent centraux. Notamment la question du genre par rapport au sexe et surtout par rapport à ce qui est l'objet spécifique de la psychanalyse, soit le sexuel infantile. Il faut en effet constamment nous rappeler, nous psychanalystes, que si nous devons accepter de tout entendre, de nous intéresser à tous les aspects de la condition humaine, cela ne veut pas dire que la psychanalyse peut discourir à propos de tout avec la même compétence. Les débats et combats autour des nouvelles questions sur le genre, l'orientation sexuelle, les nouvelles parentalités, etc. certes nous interpellent, ce qui ne veut pas dire que nous sommes autorisés à nous prononcer à leur sujet en tant qu'experts. S'il y a une position contraire à celle de l'analyste, c'est bien celle de l'expert, puisque nous devons par principe nous abstenir de savoir le bien de nos patients. La seule expertise que nous pouvons revendiquer, c'est celle qui entre dans l'établissement et le maintien du cadre et de la méthode analytique. Par ailleurs, il ne s'agit pas non plus de mettre la psychanalyse à la mode du jour; comme tu le 
soulignes, la psychanalyse ne peut sans dommage être mise au service d'une cause, aussi noble soit-elle... il nous faut donc bien veiller à ne pas dégrader la pensée psychanalytique en idéologie.

On pourrait croire que, confrontée à de nouvelles questions comme celles que je viens de nommer, la psychanalyse a cessé depuis longtemps d'apporter du nouveau. Nous ne sommes plus au temps où Freud défrichait le terrain et ouvrait des chemins jusque-là inexistants. Par ailleurs, notre pratique nous place dans une position bien particulière, puisque la prise en considération de l'inconscient sert avant tout à interroger de manière critique les positions (politiques, idéologiques, psychologiques, voire neuroscientifiques) apparemment bien fondées. La psychanalyse, comme Freud l'indique, travaille per via di levare, et ce qu'il s'agit souvent de lever, ce sont les illusions du moi individuel ou des moi réunis en collectif. Mais, puisque les psychanalystes aussi forment des collectifs aptes à se complaire dans une illusion commune, il nous faut, comme tu nous y invites, balayer d'abord devant notre propre demeure et ne pas répondre aux questions du jour par des slogans tels que déni de la castration et forclusion du nom-du-père. Heureusement, je crois que même après plus d'un siècle de psychanalyse, il y a encore lieu pour elle de revendiquer de la nouveauté, ce qui nous ramène à la centralité du refoulement. Cela parce que notre objet spécifique - la réalité psychique, le sexuel infantile - a comme particularité d'être inséparable de son refoulement et que chaque fois que ce refoulement est temporairement levé, ce qui émerge est toujours «frais comme au premier jour», pour citer les Études sur l'hystérie. S'agissant de l'inconscient, les choses se passent comme le dit la chanson: "J'ai beau t'ouvrir cent fois les bras, c'est toujours la première fois".

Tu rappelles, avec raison, que la psychanalyse ne comporte pas de Weltanschauung, pas de "vision du monde», et que le débat avec les gender studies est d'abord théorique. Il faudrait, bien entendu, pouvoir entrer dans le détail des gender studies en question; discuter par exemple de la performativité de l'assignation de genre théorisée par Judith Butler, que d'autres féministes américaines ont d'ailleurs contestée et que Butler elle-même a tenté par la suite de relativiser jusqu'à un certain point (Butler, 1993). Pour aller vite, disons que cette performativité fut d'abord présentée comme toutepuissante, capable de «formater», pour ainsi dire, le nouveau-né, presque sans reste, sans marge de manœuvre. "Pas un mot dans tout cela, écris-tu, 
sur ce qui constitue l'assignation de genre par l'inconscient des parents, soit l'objet même de la psychanalyse».

Le plus ironique est que Butler est par ailleurs censée s'inspirer de Laplanche et de la théorie des messages énigmatiques, mais sans remarquer que le message n'est énigmatique selon Laplanche que parce qu'il contient autre chose que les formes socialement reconnaissables de son contenu manifeste. Lors d'un débat tenu à Berlin en 2007 entre Laplanche et Butler, il est devenu clair que celle-ci entretenait une conception tronquée du "message» selon Laplanche, n'y retenant justement que la forme manifeste, sans doute socialement performative jusqu'à un certain point ( ¿C'est une fille! C’est un garçon!»), mais oubliant précisément ce qui fait tout l'intérêt du message énigmatique, ou compromis, selon Laplanche: c'est que la part «énigmatique» l'est non seulement pour l'infans au pôle récepteur, mais aussi pour l'adulte, émetteur malgré lui. Il y a donc beaucoup plus qu'il n'y paraît dans l'assignation de genre et, tout d'abord, il y a que la perception (elle-même culturellement influencée) du sexe anatomique ne saurait annuler les pressions exercées par les désirs inconscients des adultes, désirs qui peuvent contredire radicalement le message manifeste et qui voyagent en passagers clandestins dans la déclaration de genre, apparemment fondée sur la réalité perçue et socialement convenue. Butler parle de «gender trouble» mais ne tient pas compte du «trouble dans le message» qui le rend par le fait même beaucoup plus complexe et sujet à des efforts répétés de traduction par l'infans qui en est le récepteur. Or cela, comme je m’en expliquerai plus loin, a des conséquences fort intéressantes.

Je me permets à présent d'apporter une réflexion complémentaire dont tu me diras si elle concorde avec tes vues. Je précise tout de suite qu'en rappelant le rôle joué par l'inconscient des parents, il ne s'agit pas de remplacer la performativité des assignations manifestes par une performativité inconsciente, ce qui ne nous avancerait pas beaucoup. Par conséquent, il me semble nécessaire de revenir sur la scène de l'assignation de genre: «C'est un garçon! C'est une fille!», cela ressemble au performatif selon Austin ou à l'efficacité symbolique selon Lévi-Strauss. Mais il y a un point essentiel sur lequel il ne faut pas se méprendre. Il faut se demander sur qui opère, à ce moment inaugural, le performatif ou l'efficacité symbolique: certainement pas sur le nouveau-né, qui de toute façon n'apprendra que bien plus tard ce qu'il en est. Les sujets concernés par ce qui revient à une sorte de rituel, 
ceux sur qui opère dans un premier temps l'assignation, ce sont les parents eux-mêmes et le cercle de la famille élargie ou des amis qui les entourent. Or ceux-ci ne savent pas le tout de ce que le rituel d'assignation de genre véhicule. Si la différence anatomique est le plus souvent reconnue, cela ne la met pas à l'abri des fantasmes et théories infantiles, des désirs qui perdurent dans l'inconscient des adultes participants. C'est sur un temps plus long, et selon la modalité de l'après-coup, qu'opérera la transmission effective à l'enfant de cette assignation. Après-coup qui emprunte des chemins fort contournés, l'opération d'assignation allant de l'échographie prénatale à la couleur des murs de la chambre et incluant les usages linguistiques, vestimentaires, le choix des jouets, etc. Ces divers moments d'assignation de genre ne "performent» pas directement et mécaniquement. C'est que l'infans sur lequel porte l'assignation de genre reçoit celle-ci comme message à traduire, à interpréter. L'enfant, il faut y insister, est un traducteur né. Or Freud luimême nous a appris que traduction et refoulement sont indissociables. Bien entendu, le traducteur est bien forcé d'utiliser les outils de traduction fournis par la culture dont les parents et éducateurs sont les représentants; reste que, comme l'expérience l'atteste, et comme Freud le soulignait,

le refoulement [et donc, ajouterons-nous, la traduction] opère de façon hautement individuelle; chacun des rejetons du refoulé, pris un à un, peut avoir son destin particulier; un peu plus ou un peu moins de déformation fait que le [résultat final] vire du tout au tout. [...] les objets préférés des hommes, leurs idéaux, proviennent des mêmes expériences vécues que les objets les plus exécrés par eux, et ne se différencient les uns des autres, à l'origine, que par de minimes modifications. (Freud, 1915, p. 195)

C'est là, avant la lettre, l'énoncé du mode de fonctionnement des systèmes hypercomplexes dont la dynamique est non-linéaire et dont la multitude de variables ainsi que les nombreuses réitérations des cycles, avec un écart même minuscule survenant à chacun de ces cycles, fait en sorte que le résultat final ne sera pas parfaitement prévisible. Cela est vrai pour le système météo, où il n'y a que des forces physiques à l'œuvre. Cela l'est d'autant plus pour le système psychique où se combinent des déterminants biologiques, sociaux, culturels, familiaux, et leur traduction/refoulement par le sujet lui-même. Sans oublier les hasards des rencontres qui feront se cristalliser l'assomption d'une identité de genre plutôt qu'une autre, un choix d'objet sexuel plutôt qu'un autre. 
En passant, pour bien saisir la variété possible des résultats, nous psychanalystes avons intérêt à tenir compte de la différence qui passe entre causalité et déterminisme inconscient ${ }^{2}$. Ce dernier est indéniable, mais au contraire de l'idée de causalité, il laisse une marge d'indétermination où jouent aussi des facteurs contingents, voire aléatoires, ceux dont on ne peut discourir qu'après-coup. La force de gravité de la planète Terre certes détermine, en dernière analyse, l'effondrement d'un pont, mais elle ne le cause pas comme le fait l'explosion contingente de la dynamite qui en brise les piliers.

C'est là une très bonne nouvelle pour l'individu. Cela signifie que, en tant que réceptionnaire du message de genre, il dispose de ce fait, dans la plupart des cas, de la liberté relative du traducteur/refouleur. Liberté nullement éprouvée subjectivement, il va sans dire. Appelons cela, un peu à la manière des statisticiens, des "degrés objectifs de liberté», apparaissant au cours de processus où jouent tant les déterminismes inconscients que les déterminismes sociaux. Tenons aussi présent à l'esprit que les nombreux cycles de traduction/refoulement se produisant durant l'enfance préparent le terrain aux grandes révisions amenées par la puberté et l'adolescence - des tempêtes non seulement hormonales, mais aussi familiales et sociales qui conduiront à terme à des résultats hautement individuels et dont tu rappelles qu'ils ne sont pas exempts de révisions ultérieures. L'après-coup, que tu as toi-même bien étudié, est la forme temporelle inséparable de l'opération de traduction/refoulement et il ne cesse jamais d'opérer.

Le point de vue individuel, résultat après coup de la rencontre entre les divers déterminants et la capacité traductive du sujet - point de vue de ce que je nommerais la «subjectalité» pour ne pas la confondre avec le subjectivisme -, c'est justement celui qui me semble gravement absent de la réflexion de Judith Butler. Cela parce que Butler, qui pourtant convoque Laplanche, manque à tenir compte, d'une part, comme déjà dit, de la composante inconsciente qui voyage en clandestin à l'intérieur du message d'assignation, et, d'autre part, de l'œuvre de traduction/refoulement de l'enfant qui fait l'objet de cette assignation. Pourtant Laplanche est assez clair là-dessus lorsqu'il écrit que le sexuel infantile (qu'il nomme "Sexual», pour éviter tout malentendu) est ce qui reste de la symbolisation/refoulement du genre par le sexe. J'y reviendrai. Pour l'instant retenons que la traduction/refoulement du message d'assignation opère grâce à l'outil de traduction que fournit la perception. Cette perception est dans un premier temps celle des parents, mais un jour où l'autre entrera en scène celle de l'enfant lui-même, lorsqu'il sera en mesure de constater la différence anatomique entre les sexes. Sauf 
que, comme tu le soulignes, cette perception n'est jamais pure. Elle ne sera donc pas décisive par elle-même, puisqu'elle affrontera à son tour l'énigme de la différence anatomique et que l'enfant-traducteur aura bien des raisons de la lier à toutes sortes d'autres expériences énigmatiques (sevrage, bruits dans la chambre des parents) dont il se sera fait des «théories» justement nommées par Freud «théories sexuelles infantiles». Il faut ici, me semblet-il, insister sur la maxime que tu énonces: "L'anatomie imaginaire, c'est le destin», et cette anatomie imaginaire ne surgit pas du néant, mais d'un travail compliqué fait par l'enfant-interprète. Les parents, pour leur part, n'opèrent pas différemment: leurs propres théories sexuelles infantiles, leurs propres désirs inconscients influent à leur insu sur l'assignation de genre considérée en son entier. Nous nous retrouvons ainsi constamment dans ce que l'on peut appeler un chiasme: des deux côtés, adulte et enfant, c'est le sexuel infantile qui préside au destin en question, mais cela - il vaut la peine d'y insister - en tant que déterminant et non en tant que cause.

Tu écris que l'inconscient est "politiquement incorrect», dans le sens où « il n'y a aucune chance de rencontrer dans l'inconscient une quelconque égalité entre les sexes ou les orientations sexuelles». Je crois utile de préciser, même si c'est implicite dans ton texte, que tu énonces par là un constat et non un programme politique anti-féministe. Ce constat rappelle que l'inconscient est fait, par définition, de ce qui ne peut être porté à la conscience ou intégré dans le moi par la traduction/théorisation du sujet, et qui par conséquent reste marqué des caractéristiques propres à l'infantile: son caractère pulsionnel et auto-érotique et son rapport à l'objet partiel, sans la réciprocité et la reconnaissance de l'autre comme sujet, c'est-à-dire comme objet total. Le sexuel infantile ainsi marqué par le refoulement est par définition rebelle à toute mise en ordre. Il m'apparaît que tout programme qui, au nom de la parité ou égalité entre les genres et/ou les sexes, nierait cette réalité psychique, se trouverait d'une part à confondre celle-ci et la réalité sociale et, d'autre part, aboutirait à l'exact opposé de ce que sont censées viser les théories critiques du genre. Je m'explique.

Comme je viens de l'indiquer, l'originalité de la configuration sexuelle individuelle ne saurait s'établir par décret. La critique, évidemment justifiée, de l'inégalité sociale selon le genre ne justifie pas de négliger, encore moins de nier, le poids de la réalité psychique, dont nous avons vu qu’elle ne résulte 
pas d'une intériorisation directe des valeurs dominantes, mais d'une traduction/refoulement. Butler se veut subversive sur le plan social en cherchant à abolir les catégories de genre, mais elle oublie de convoquer la source même de toute indocilité, soit le sexuel inconscient. Ce faisant, elle méconnaît ce qui fait de chaque individu un être potentiellement anti-conformiste: la fonction traductive de la psyché individuelle. Il y a ici un sérieux paradoxe: s'attaquer au langage normatif, c'est ne s'attaquer qu'à la surface des choses et, pire encore, c'est chercher à imposer une nouvelle norme sociale.

Signaler ce paradoxe, cela ne revient pas à nier la nécessité du débat et du combat social et politique, bien au contraire. Pour ce qui concerne la psychanalyse, les analystes-femmes ont, du vivant même de Freud, commencé à tenir ce débat et j'ai été récemment frappé de constater que ce débat, aux allures socio-politiques, portait en fait sur l'amalgame opéré - y compris par Freud - entre la théorétique psychanalytique et les théories sexuelles infantiles! Ainsi, dès 1926, Karen Horney dressait sur deux colonnes un tableau comparatif entre les théories psychanalytiques officielles concernant les filles et les théories sexuelles infantiles du petit garçon et montrait que la concordance était quasi parfaite (Horney, 1926). Autrement dit, les théories sexuelles infantiles étaient admises presque telles quelles en tant que théories à prétention scientifique! Les théoriciens du genre font aujourd'hui l'erreur symétrique: celle de donner tout le pouvoir au manifeste. Dans les deux cas, ce qui est perdu de vue est le rôle décisif de l'infans-traducteur, autrement dit, du processus de symbolisation/refoulement. Celui-ci d'une part - sur le versant symbolisation-traduction - produit les théories sexuelles infantiles appelées à déterminer la vie fantasmatique, les "choix» d'objet sexuel et d'identité de genre; mais d'autre part - sur le versant refoulant - laisse un résidu qui est le Sexual, écharde irritative désormais installée à demeure, source interne du trouble et instigateur de maintes révisions survenant au hasard des rencontres que la vie réserve au sujet.

Laplanche a proposé une formule concernant l'émergence de ce sexuel inconscient: "Le Sexual, écrit-il, c'est le résidu inconscient de la traductionsymbolisation du genre par le sexe.» (Laplanche, 2007, p. 153) La formule peut sembler cryptique et Laplanche déploie un long texte à sa suite afin de l'expliciter. En peu de mots, le genre est de l'ordre du message manifeste: «C'est un garçon!» ou «C'est une fille!», mais véhicule à l'insu de l'émetteur bien plus que ce que la déclaration prétend établir. Le manifeste est compromis, "contaminé» pour ainsi dire, par le Sexual parental. L'infans qui reçoit le message manifeste est exposé à un surplus d'excitation, à un 
trouble résultant de la disparité sexuelle fondamentale entre adulte et enfant. Dans la mesure où cet enfant n'est pas soumis à une imposition violente - l'intromission décrite par Laplanche (1990) - qui paralyse ses moyens individuels de traduction, sa psyché se chargera de donner un sens à ce qui trouble l'apparente clarté de l'assignation de genre. Avec les éléments puisés dans l'entourage (imaginaire familial et social) additionnés à ceux de l'imaginaire naissant de son expérience du corps érogène, il finira par produire sa propre traduction-symbolisation intime: du genre assigné on parvient ainsi au genre assumé. Mais cette assomption du genre laissera forcément un reste de sexuel infantile avec son caractère essentiellement pulsionnel, c'est-à-dire rebelle à toute mise en ordre. Ce sexuel infantile, ou Sexual, exercera une pression constante pour de nouvelles traductions qui seront du même coup autant de refoulements successifs, dans un processus sans fin par lequel la configuration psycho-sexuelle individuelle se renégociera selon les nécessités du moment. Le genre assumé, bien que déterminé en bonne part par le social, l'éducation et l'implantation du sexuel parental, bénéficie donc d'une relative liberté d'interprétation de la part du sujet. C'est ainsi que le masculin comme le féminin admettent de nombreuses nuances individuelles. Et il va de soi que le résultat est rarement, si jamais, exempt d'ambiguïtés, d'ambivalences et de potentialités inexplorées. Tout système vivant est, selon Gilbert Simondon (1958), par définition méta-stable, c'est-à-dire susceptible d'évoluer. Cela à moins qu'un trauma significatif, telle l'intromission déjà signalée, n’ait immobilisé le processus traductifs, fixant alors le résultat en un scénario difficile sinon impossible à re-traduire/re-symboliser.

Cette dernière éventualité, je crois que c'est à elle que s'adresse à son insu la théorie critique des genres. En effet, on pourrait avancer que les normes sociales, les rôles traditionnellement réservés à l'un et l'autre genre, les inégalités qui en résultent, ne sont pas sans opérer une «fixation» collective sur une forme particulière, sur une traduction plus ou moins rigidement normative, sur une symbolique dominante qui informe en retour les traductions individuelles, formant un cercle vicieux qui tend à la reproduction du même.

Ainsi, certaines des théories sexuelles infantiles peuvent être, et sont dans les faits, sélectionnées (au sens darwinien) par les processus sociaux quand elles font l'affaire, par exemple, du maintien de l'inégalité homme-femme au plan social, économique et politique. La théorie infantile selon laquelle il n'y a qu'un seul sexe, le phallique, dont la petite fille est dépourvue, est bien faite pour travailler de manière inconsciente à maintenir la division sexuelle du travail instaurée depuis la nuit des temps. Mais cette théorie sexuelle 
infantile est appelée à être dépassée/conservée, entre autres grâce à un travail de psychanalyse, par la reconnaissance d'une différence fondée sur l'existence, au lieu de l'être castré, d'un autre sexe. Dépassement allant à l'encontre de la théorie de l'unisexualité phallique qui ne voit dans le sexe femelle que manque et castration. La théorie infantile ne disparaît pas pour autant (d'où l'expression «dépassée/conservée»), mais une tension productive se sera établie chez le sujet qui l'aura dépassée en direction d'une symbolisation plus fluide. Par ailleurs, prendre acte de la présence de cette théorie infantile ne contredit nullement, bien au contraire, la nécessité de la lutte pour l'égalité sociale, économique et politique, des sexes et des genres. Elle dit seulement qu'il n'y aura pas de victoire définitive, comme c'est aussi le cas de la lutte pour la démocratie, ainsi que la conjoncture présente nous le montre.

Ton constat, en toute impartialité, du sexuel infantile comme étant «politiquement incorrect» me semble donc opérer sur deux plans à la fois. Au plan social, où loin d'être en soi politiquement conservatrice ou réactionnaire, la théorie psychanalytique de l'inconscient informe au contraire la collectivité de la persistance en son sein du sexuel infantile qui, pour indélogeable qu'il soit de l'inconscient individuel, ne justifie aucunement l'infantilisme sexuel comme principe organisateur de la société, mais permet au contraire d'identifier les sources de cet infantilisme social et de le dénoncer là où il persiste. Au plan individuel, où la psychanalyse nous enseigne que l'organisation psychique ne se décrète pas. Voire, que toute tentative de la mettre au pas ne peut être que contre-productive et contredit radicalement l'intention de départ qui serait, tâche par ailleurs impossible, de libérer la vie sexuelle de toute contrainte. Face aux déterminants sociaux, le psychisme individuel ne peut que se constituer, se vectoriser en une résultante originale, combinant tous les déterminants - parentaux, familiaux, sexuels inconscients - à l'imprévu, aux hasards d'une vie.

En terminant, je note que si nous assistons aujourd'hui à l'émergence de nouvelles variantes dans les configurations sexuelles manifestes, c'est dans la mesure où des facteurs nouveaux, allant des possibilités technologiques (technologies médicales et technologie des communications notamment) aux libertés politiques et sociales, ont ouvert la voie à des formes d'expression qui n'étaient pas nécessairement toutes réprimées ou refoulées auparavant, mais, pour plusieurs d'entre elles, proprement inexistantes avant que les nouveaux instruments sociaux ne soient disponibles et ne modifient par conséquent l'imaginaire social. Comme le disait Marx, la société ne se pose que des questions qu'elle a les moyens de résoudre. Cela n'autorise toutefois 
aucunement à ignorer l'autre volet, celui du sexuel inconscient. Les configurations individuelles, que ce soit l'assomption de genre, le choix d'objet sexuel voire de carrière professionnelle, seront toujours des compromis entre les formes socialement proposées et le processus de traduction/refoulement d'un sexuel infantile appelé à se répéter sans cesse. Cet inépuisable processus visant à traduire le Sexual - l'inconscient, le sexuel infantile - peut jouer sur deux tableaux: d'une part il peut, comme on l'a vu, donner lieu à des fixations que le social sélectionnera, renforcera et mettra à profit selon ses propres logiques (maintien de la disparité de genre, par exemple), d'autre part il peut être à la source des solutions nouvelles, voire provocantes pour l'ordre établi, mais en tout cas individuellement élaborées en fonction des déterminismes internes et externes. La psychanalyse se tient là, au point de rencontre ou de séparation entre ces deux types de destin, assistant le sujet dans son effort de symbolisation, mais vérifiant chaque fois que ce qui est un déterminant inconscient peut en même temps, et pour les mêmes raisons, être à la source de l'intime liberté du sujet.

Dominique Scarfone dscarfone@gmail.com

\section{Notes}

1. Commentaire prononcé à la suite de la conférence de Jacques André au Colloque de la revue Filigrane, le 2 novembre 2018 à Montréal.

2. Assez tôt, dans l'histoire de la psychanalyse, s'est installée une confusion entre déterminisme et causalité, comme l'atteste le commentaire d'un physicien du nom de Markus Reiner, écrit sur invitation du Psychoanalytic Quarterly et publié en anglais dans le tout premier numéro de la revue (Reiner, 1932).

\section{Références}

Butler, J. (1993). Bodies that Matter. On the Discursive Limits of "sex". Oxon et New York: Routledge.

Freud, S. (1915). Le refoulement. Dans Euvres complètes XIII. Paris: Presses universitaires de France.

Horney, K. (1926). The Flight from Womanhood: The Masculinity-Complex in Women, as Viewed by Men and by Women, International Journal of Psychoanalysis., 7, 324-339.

Laplanche, J. (1990). Implantation, intromission. Dans Le primat de l'autre en psychanalyse. Paris: Flammarion, 1997.

Laplanche, J. (2007). Sexual. La sexualité élargie au sens freudien. Paris: Presses universitaires de France.

Reiner, M. (1932). Causality and Psychoanalysis - A Letter to the Editors of the Psychoanalytic Quarterly. Psychoanalytical Quarterly, 1, 701-714.

Simondon, G. (1958). L'individuation psychique et collective. Paris: Aubier, 2007. 